Schweiz. Gastroenterol. 2020 - 1:79-85 https://doi.org/10.1007/s43472-020-00018-8 Publié en ligne: 21 . Septembre 2020 (c) The Author(s) 2020

\section{Lucia Zampaglione ${ }^{1,2}$ - Jacopo Ferrari ${ }^{1}$ Nicolas Goossens ${ }^{2}$}

'Vita-Salute San Raffaele University, Milan, Italie

${ }^{2}$ Service de Gastroentérologie et Hépatologie, Hôpitaux Universitaires de Genève, Genève, Suisse

\title{
La stéatopathie dysmétabolique
}

\section{Introduction}

La stéatopathie dysmétabolique (ou NAFLD: non-alcoholic fatty liver disease) fait référence au spectre des maladies caractérisées par une infiltration de graisses dans les hépatocytes, et ce après exclusion des autres causes secondaires de stéatose (ex: une consommation significative d'alcool) [1]. La NAFLD recouvre un spectre d'anomalies pathologiques. La majorité des patients nont qu'une simple stéatose hépatique sans inflammation ni fibrose hépatique (NAFL), cependant, on estime qu'entre 7 à $30 \%$ des patients développeront une stéatohépatite non alcoolique (ou NASH: non-alcoholic steatohepatitis), et au moins un tiers de ces derniers cas vont progresser vers une fibrose et cirrhose [2]. Dans cet article, nous allons aborder les nouvelles définitions proposées de la stéatopathie dysmétabolique, des données épidémiologiques récentes, la progression de la maladie, sa prise en charge, ainsi que les perspectives futures importantes.

\section{MAFLD ou NAFLD: un débat sur la nouvelle définition proposée}

La définition actuelle de la NAFLD est basée sur la présence de stéatose dans plus de $5 \%$ des hépatocytes ainsi qu'une exclusion de toute autre cause d'accumulation secondaire de graisses dans le foie, en particulier une consommation d'alcool excessive ou les hépatites virales [3]. Cependant, cette définition ne correspond pas vraiment à nos connaissances actuelles de la pathogénèse de cette maladie, qui est fortement liée au dysfonctionnement métabolique. De plus, la définition actuelle de la NAFLD exclue une certaine catégorie de patients cirrhotiques, lorsque ceux-ci ne présentent plus de stéatose, malgré qu'ils aient plusieurs facteurs de risque connus de la NAFLD. Chez ces patients, la preuve histologique de la stéatose, l'inflammation ou les lésions hépatocellulaires classiques sont souvent remplacées par d'abondantes travées fibreuses qui peuvent masquer l'origine de l'hépatopathie. Ces patients seraient plus exactement classés à un stade avancé sur un spectre élargi de la NAFLD, compte tenu des mécanismes moléculaires sous-jacents mis en jeu. A la lumière de cette nomenclature insuffisante, un groupe d'experts a récemment proposé un nouveau terme, «MAFLD», ou metabolic associated fatty liver disease, qui reflète plus justement la pathogénèse et l'histoire naturelle de la maladie, en visant une amélioration de la stratification et de la prise en charge de ces patients [4]. Cette définition, permet un diagnostic positif, en se basant sur la preuve histologique, l'imagerie ou les biomarqueurs sériques de stéatose hépatique, accompagné d'un des trois critères suivants: le surpoids/obésité, la présence d'un diabète de type 2 ou une évidence de dysrégulation métabolique. La dysrégulation métabolique, ici, est défini par la présence de deux (ou plus) caractéristiques associées au syndrome métabolique. Ce changement de terminologie offre un avantage clinique important en changeant les critères diagnostics, d'un diagnostic négatif (basé sur l'exclusion de toutes les autres pathologies hépatiques) vers un diagnostic positif (basé sur la présence de critères d'inclusion). Ceci est important, compte tenu de l'incidence croissante de la NAFLD/MAFLD, qu'on retrouve souvent en association avec d'autres causes d'hépatopathies, comme l'alcool ou les hépatites virales permettant ainsi de poser un double (ou triple ...) diagnostic avec des critères positifs de la MAFLD. Par ailleurs cette approche permettrait aux patients cirrhotiques sans stéatose identifiée qui remplissent les critères diagnostic proposés de la MAFLD, d’être considérés comme MAFLD associée à une cirrhose, et d'éviter le terme ambiguë de "cirrhose cryptogénique». En effet, dans une étude cas-témoins qui avait pour objectif de déterminer quelle partie des cas de cirrhose cryptogénique était associée avec une dysfonction métabolique sous-jacente, les chercheurs ont trouvé que la prévalence de l'obésité ( $55 \%$ vs. $24 \%$ ) et celle du diabète type 2 ( $47 \%$ vs. $22 \%$ ) étaient significativement plus élevées chez les patients avec une cirrhose cryptogénique comparé aux témoins [5]. Par conséquent, le changement de définition va permettre de catégoriser plus précisément ces patients cirrhotiques.

Alors que le terme MAFLD reflète probablement une définition plus précise de cette pathologie hépatique et amène de nombreux avantages, certains auteurs sont plus prudents et proposent de discuter plus largement ce changement de terminologie [6]. Ils soulignent notamment que cette nouvelle définition garde une certaine ambiguïté dans la mesure où il $\mathrm{y}$ a un manque de consensus général sur les critères de «santé métabolique». Par ailleurs ils craignent que ce changement de terminologie rajoute de la confusion aux dépens des sujets particulièrement à risque en particulier ceux à risque de stade avancé de la maladie. De plus, ces auteurs argumentent que le changement de nomenclature doit accompagner des progrès significatifs dans notre compréhension de la pathogénèse et les bases moléculaires de cette maladie, qui sont clairement encore insuffisamment compris. En pratique, le plus 


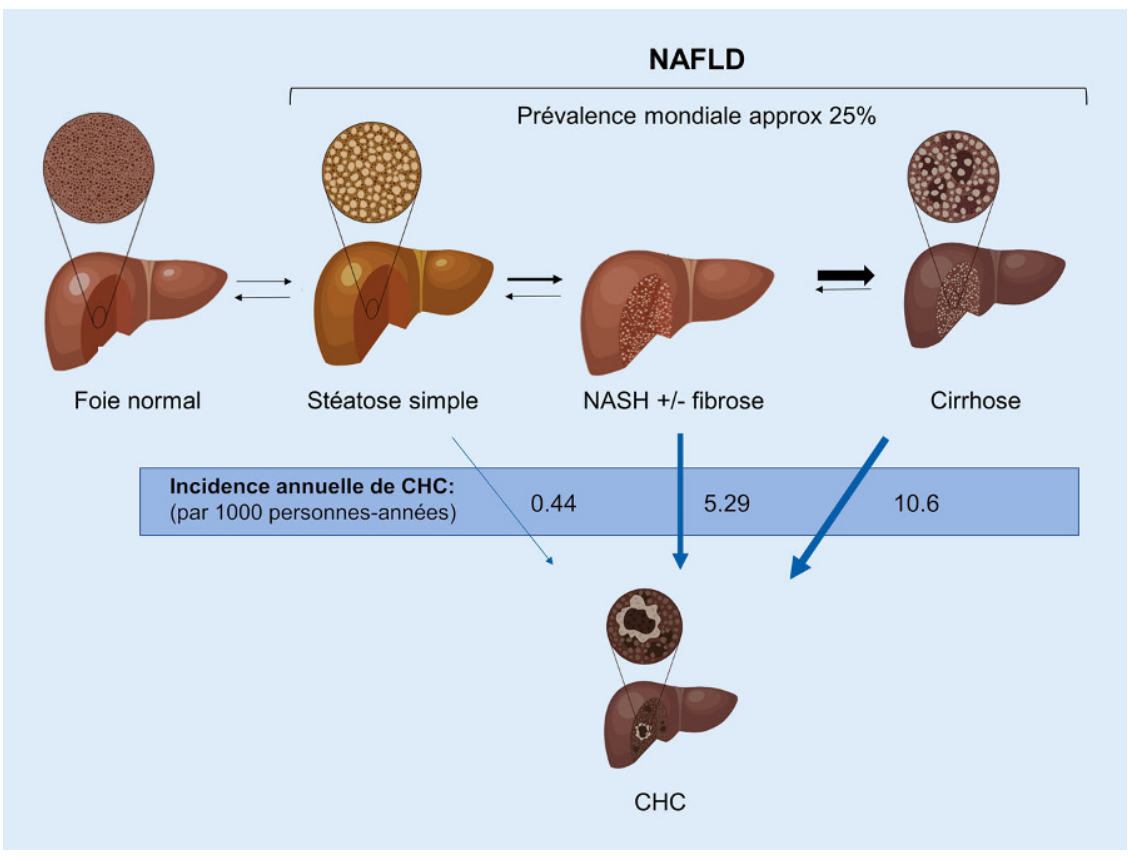

Fig. $1 \Delta$ Histoire naturelle de la NAFLD (non-alcoholic fatty liver disease). Les estimations de la prévalence de la NAFLD et de l'incidence annuelle de carcinome hépatocellulaire $(\mathrm{CHC})$ sont tirées de Younossi et al. [35] saufl'incidence annuelle de $\mathrm{CHC}$ chez les cirrhotiques qui provient de Kanwal et al. [36]. NASH non-alcoholic steatohepatitis

important, quelle que soit la nomenclature utilisée, reste que le patient demeure au centre de la prise en charge et que ces pathologies complexes puissent continuer à bénéficier d'une approche multidisciplinaire comme discuté ci-dessous.

\section{Epidémiologie et association avec le syndrome métabolique}

La NAFLD est la pathologie hépatique la plus fréquente dans le monde, affectant environ $25 \%$ de la population générale [7]. Elle est plus fréquente dans les pays industrialisés Occidentaux, le Moyen-Orient et l'Amérique du sud où les facteurs de risque majeurs comme le syndrome métabolique, le diabète type 2 et lobésité androïde sont fréquents [7-10]. La NAFLD représente également un poids économique significatif. Par exemple, aux Etats-Unis d'Amérique, on estime à plus de 64 millions de personnes avec une NAFLD avec un coût annuel de soins aux environs de 103 milliards USD [11]. Dans 4 pays importants d'Europe, l'Allemagne, la France, l'Italie et le Royaume-Uni, on estime que 52 millions de personnes ont une NAFLD, correspondant à un coût annuel de soins de 35 milliards d'euros (allant de $354 €$ à $1163 €$ par patient) [11]. A des fins de comparaison, le CDC (Centers for Disease Control) aux Etats-Unis rapporte que l'accident vasculaire cérébral coute environ 34 milliards de USD annuellement [12]. Plus inquiétant encore, la prévalence de la NAFLD est en hausse et ce de manière rapide. Dans une étude de la NHANES (National Health and Nutrition Examination Survey) aux Etats-Unis, une cohorte sétendant sur 30 ans, les chercheurs ont trouvé que la prévalence de la NAFLD a significativement augmenté, affectant à peu près $20 \%$ de la population générale entre 1988 et 1994 et atteignant 31,9\% entre 2013 et 2016 [13]. Cela contraste nettement avec les autres pathologies chroniques du foie comme l'hépatopathie alcoolique et l'hépatite $B$ qui sont restées stables, et l'hépatite $\mathrm{C}$ qui a diminué d'environ $50 \%$. En effet, durant les 30 dernières années aux EtatsUnis, la NAFLD a été la seule pathologie hépatique avec une prévalence en hausse. Dans cette analyse, l'augmentation de l'obésité et le diabète de type 2 dans la population étaient le prédicteur majeur de la hausse de la NAFLD. Compte tenu de la forte association entre le diabète type 2 et la NAFLD, une étude récente visait à démontrer la relation entre ces deux pathologies [14]. Les chercheurs ont examiné 80 différentes études sur 20 pays, incluant 49.419 patients. Ils ont trouvé que $55,5 \%$ des patients diabétiques de type 2 sont affectés par la NAFLD. En Europe, cette prévalence est encore plus élevée, avec $68 \%$ des patients diabétiques type 2 qui présentent une NAFLD associée. Par ailleurs, la prévalence globale estimée de la NASH chez les patients atteints de diabète de type 2 est de $37 \%$, significativement plus élevée que celle trouvée dans la population générale et $17 \%$ des patients atteints de NAFLD et de diabète de type 2 ayant eu une biopsie hépatique avaient une fibrose avancée.

La forte association entre la NAFLD et l'obésité a poussé à la création de modèles pour prédire la prévalence et l'augmentation de la NAFLD à l'échelle d'une population basés sur l'évolution de l'incidence et prévalence de l'obésité. En Suisse, cette approche a été utilisée pour modéliser lévolution épidémiologique de la NAFLD jusqu'en 2030 [15]. Basé sur ces modèles, la prévalence de la NASH devrait augmenter de $40 \%$ entre 2018 et 2030 et le nombre de décès est prédit à la hausse de 580 décès en 2018 à 820 décès en 2030. Tandis que ces chiffres ne sont que des estimations et restent encore à être confirmés, l'épidémie croissante de l'obésité, l'augmentation de l'âge des résidents suisse en association avec des taux élevés de diabète type 2 , supportent tous la prédiction de l'augmentation du poids épidémiologique de cette pathologie et de la mortalité associée à la NAFLD et la NASH.

\section{Histoire naturelle et progression}

La NAFLD est une pathologie à progression lente dont l'histoire naturelle reste toujours incomplètement élucidée, car peu d'études de haute qualité ont été conduites (• Fig. 1). Dans une récente cohorte prospective de suivi des biopsies hépatiques, 129 patients inclus entre 1988 à 1993 étaient reconvoqués à 2 reprises (13,7 et 9,3 années d'intervalles respectivement), $34 \%$ avaient développés une fibrose avancée et 9,3\% des patients une hépatopathie au stade terminal [16]. Ces 
Résumé · Zusammenfassung · Riassunto

Schweiz. Gastroenterol. 2020 1:79-85 https://doi.org/10.1007/s43472-020-00018-8

(c) The Author(s) 2020

\section{Zampaglione $\cdot$ J. Ferrari $\cdot$ N. Goossens}

\section{La stéatopathie dysmétabolique}

\section{Résumé}

La stéatopathie dysmétabolique (NAFLD) est une maladie hépatique à évolution lente qui comprend un spectre allant de la stéatose hépatique non alcoolique, à la stéatohépatite non alcoolique (NASH) et dans les cas graves, la cirrhose. La NAFLD est la pathologie hépatique la plus répandue dans les pays occidentaux industrialisés, dont la Suisse, avec une prévalence en croissance rapide reflétant celle de l'obésité et du diabète de type 2 . Un panel d'experts a récemment proposé l'utilisation d'une nomenclature alternative, la stéatose hépatique associée à un dysfonctionnement métabolique (MAFLD) dont l'utilisation reste discutée. Bien qu'il existe un pipeline de développement de médicaments très actif et que de nombreux médicaments sont actuellement en essai clinique de phase II et III, aucun traitement pharmacologique n'a encore été approuvé pour la NASH. Dans certains cas une chirurgie bariatrique pourra être discutée. Néanmoins, à l'heure actuelle, la prise en charge reste centrée sur les mesures hygiéno-diététiques et le suivi par le médecin de premier recours ou le spécialiste en fonction du stade d'hépatopathie et des comorbidités.

Mots clés

Cirrhose · Foie gras · Diabète · Stéatose . Carcinome hépatocellulaire

\section{Die nicht-alkoholische Fettlebererkrankung}

\section{Zusammenfassung}

Die nicht-alkoholische Fettlebererkrankung (NAFLD) entwickelt sich schleichend und umfasst ein Spektrum, das von der einfachen nicht-alkoholischen Fettleber bis zur nichtalkoholischen Steatohepatitis (NASH) und in schweren Fällen bis zur Leberzirrhose geht. Die NAFLD ist die häufigste Lebererkrankung in westlichen Industriestaaten inklusive der Schweiz; ihre rasch zunehmende Prävalenz entspricht dem Anstieg der Adipositas und des Typ-2-Diabetes. Ein Expertenpanel hat die Umbenennung in «metabolisch assoziierte Fettlebererkrankung» (MAFLD) vorgeschlagen, was derzeit noch Gegenstand von Diskussionen ist. Obwohl sehr aktiv an de Entwicklung von Medikamenten gearbeitet wird und zahlreiche Medikamente aktuell in Studien der Phasen 2 und 3 geprüft werden, ist bisher noch keine medikamentöse Therapie zur Behandlung der NASH zugelassen. In manchen Fällen kann ein bariatrischer Eingriff erwogen werden. Dennoch konzentriert sich die Behandlung bisher vor allem auf Änderungen des Lebensstils und auf die Langzeitbetreuung durch den Allgemeinmediziner oder Spezialisten je nach Stadium der Lebererkrankung und vorhandenen Begleiterkrankungen.

Schlüsselwörter

Leberzirrhose - Fettleber · Diabetes · Steatose . Hepatozelluläres Karzinom

\section{La malattia del fegato grasso non alcolico}

\section{Riassunto}

La malattia del fegato grasso non alcolico (NAFLD/Non-alcoholic fatty Liver Disease) si sviluppa in modo insidioso e copre uno spettro che va dal semplice fegato grasso non alcolico alla steatoepatite non alcolica (NASH/Non-Alcoholic Steatohepatitis) e nei casi più gravi alla cirrosi epatica. La NAFLD è la malattia epatica più comune nei paesi industrializzati occidentali, inclusa la Svizzera; la sua prevalenza, in rapido aumento, corrisponde all'aumento dell'obesità e del diabete di tipo 2. Un comitato di esperti ha recentemente proposto di ribattezzarla «malattia del fegato grasso associata a disfunzione metabolica» (MAFLD/Metabolic Associated Fatty Liver Disease Metabolic-associated fatty liver disease), questione che attualmente è ancora oggetto di discussione. Sebbene si lavori attivamente allo sviluppo di farmaci e numerosi medicinali siano attualmente in fase di sperimentazione in studi di fase 2 e 3, non è stata ancora approvata alcuna terapia farmacologica per il trattamento della NASH. In alcuni casi può essere presa in considerazione la chirurgia bariatrica. Tuttavia, finora il trattamento si concentra principalmente su cambiamenti dello stile di vita e sull'assistenza a lungo termine da parte di un medico di base o di uno specialista, a seconda dello stadio della malattia epatica e delle malattie concomitanti esistenti.

\section{Parole chiavi}

Cirrosi epatica $\cdot$ Fegato grasso $\cdot$ Diabete

Steatosi $\cdot$ Carcinoma epatocellulare résultats suggèrent qu'en allongeant les périodes des biopsies de suivi pour permettre une évaluation précise de l'histoire naturelle, la NAFLD semble avoir un pire pronostic que ce qui a été rapporté précédemment. Dans cette étude le suivi moyen a été de 19,8 ans, et la progression vers la fibrose avec un stade $\geq 1$ a été observé chez $61 \%$ des patients [16]. La présence d'un diabète de type 2 a été associée avec un risque élevé d'évolution vers une hépatopathie de stade terminal (33\% contre $8,5 \%, p=0,026$ ). Dans une méta-analyse de biopsies appariées (paired biopsy) ayant pour but de déterminer la différence de progression de la fibrose chez les patients avec une NAFLD et ceux de la NASH, des chercheurs ont ana- lysé les biopsies de 411 patients collectées à un an d'intervalle. La durée moyenne de progression d'un stade de fibrose était de 7,1 années chez les patients avec une NASH mais 14,3 années pour les sujets avec NAFL [17]. La progression lente mais persistante de cette maladie a de fortes répercussions pour le clinicien car la maladie est réversible jusqu’à certains 


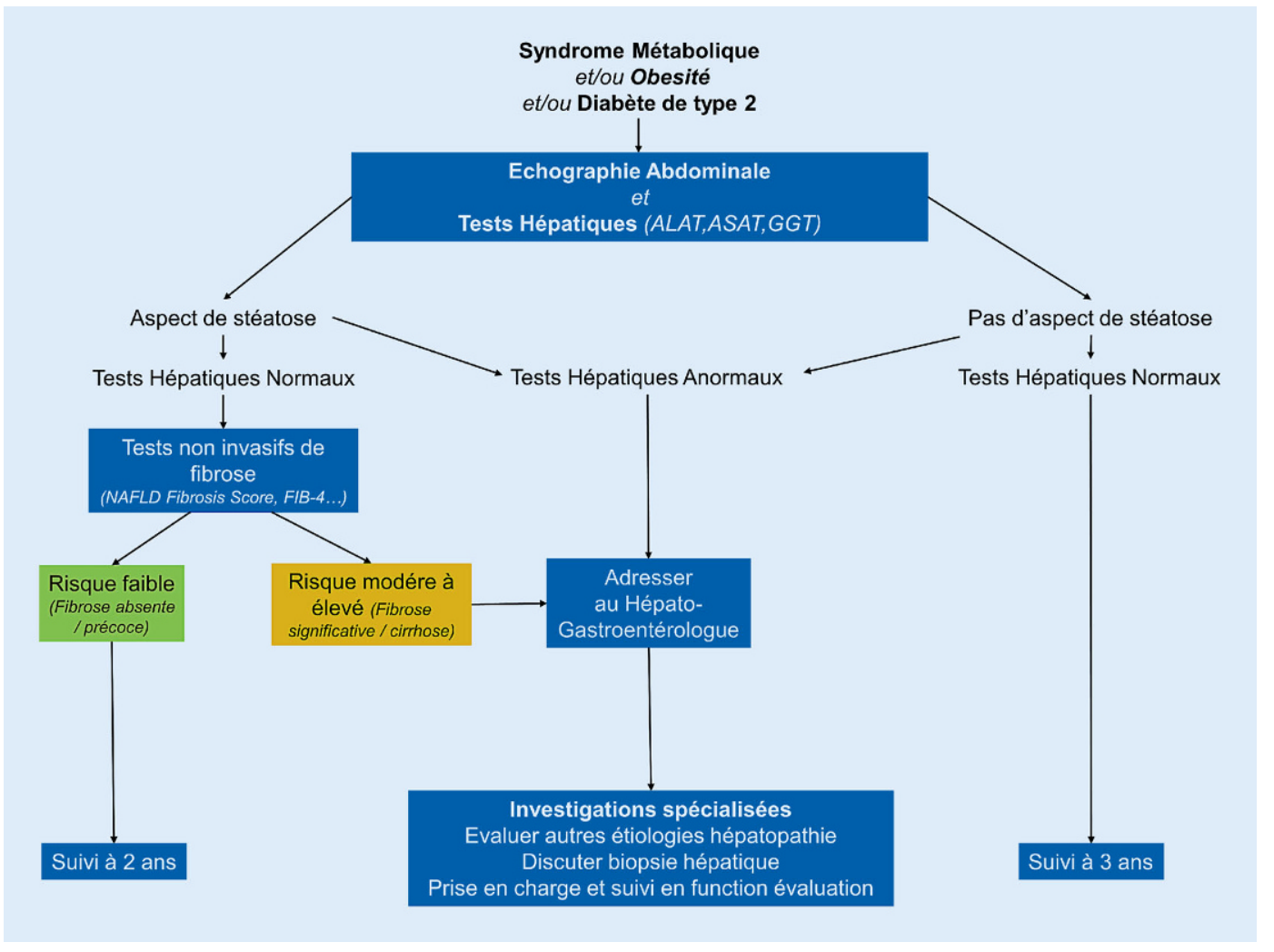

Fig. $2 \triangleleft$ Recommandations de dépistage et prise en charge de la NAFLD (non-alcoholic fatty liver disease). Adapté des recommandations européennes [3]. ALAT alanine aminotransférase, ASAT aspartate aminotransférase, GGT gamma glutamyl-transférase stades tardifs et les interventions pour inverser une telle fibrose avant d'atteindre un stade irréversible sont impératives.

\section{Dépistage et diagnostic de la NAFLD}

Les recommandations récentes proposent de rechercher une stéatose hépatique chez tous sujets à risque de NAFLD, en particulier ceux avec des facteurs de risque métaboliques ou un syndrome métabolique (• Fig. 2). La biopsie hépatique reste le gold standard (imparfait) pour le diagnostic de la NAFLD, cependant, vu lépidémiologie croissante il est souvent nécessaire de se baser sur un diagnostic basé sur des données cliniques, des examens paracliniques (perturbation des enzymes hépatiques, scores cliniques) et les examens d'imagerie (l'échographie et lélastométrie hépatique) tout en éliminant les autres causes de pathologie hépatiques. Pour les patients dont le diagnostic reste incertain après une évaluation noninvasive, une biopsie hépatique reste indiquée comme suggéré dans les recommandations Européennes [3]. En plus de la documentation de la stéatose hépatique, la surveillance de la fibrose est importante car elle reste un facteur pronostique défavorable tant général que spécifique au foie dans la NAFLD [18]. Chez les sujets avec une fibrose faible à modérée le clinicien privilégiera un diagnostic non-invasif, alors que chez les sujets avec suspicion d'hépatopathie avancée voire une cirrhose, une biopsie hépatique reste souvent indiquée pour confirmer le diagnostic et évaluer le rôle des cofacteurs éventuels [19].

\section{Prise en charge}

La NAFLD/NASH est une pathologie très hétérogène avec une physiopathologie complexe et multifactorielle. Certains mécanismes clés incluent l'insulinorésistance, les facteurs liés au mode de vie, la leptine, le stress oxydatif, les bactéries intestinales et des facteurs héréditaires [20]. Compte tenu du large éventail de facteurs mis en jeu, ce nest pas surprenant que la majorité des essais thérapeutiques menés pour la NASH ont échoué ou bien ont montré un effet très modeste.
Jusqu'à ce jour, il n'existe aucun traitement pharmacologique approuvé ciblé contre la NASH. La pierre angulaire du traitement pour la NASH reste les mesures hygiéno-diététiques, et en particulier la perte de poids pour les patients avec un index de masse corporelle $\geq 25 \mathrm{~kg} / \mathrm{m}^{2}$. A travers de plusieurs études, la perte de poids a été impliquée comme facteur clé pour l'amélioration de l'histologie du foie, des tests hépatiques, ainsi que la qualité de vie [21,22]. Par exemple, dans une des plus larges études prospectives conduites à ce jour à Cuba, 293 patients avec une NASH histologiquement documentée ont subi une période de 52 semaines de mesures hygiéno-diététiques avec un régime hypocalorique et augmentation de l'exercice physique [23]. $25 \%$ de ces patients ont démontré une résolution de la stéatohépatite, tandis que $19 \%$ ont eu une régression de la fibrose. Le degré de la perte de poids était indépendamment associé avec l'amélioration de tous les paramètres histologiques; chez les patients avec une perte pondérale $\geq 5 \%$ de leur poids corporel, on observait une résolution de la NASH chez $58 \%$ alors que chez les patients qui ont perdu $\geq 10 \%$ de 
leur poids corporel, $90 \%$ ont eu une résolution de la NASH et $45 \%$ montraient une régression de la fibrose. Malheureusement, seule une minorité des patients de cette étude $(30 \%)$ ont pu avoir une perte pondérale significative à travers les mesures hygiéno-diététiques seules et encore moins ont pu maintenir cette perte de poids à long terme.

La principale cause de décès chez les sujets NAFLD sont les maladies cardiovasculaires [24]; en fait, la NAFLD représente un facteur de risque indépendant de maladies cardio-vasculaires [25]. Il n'est donc pas surprenant que, le régime méditerranéen, bien connus pour ses bienfaits chez les patients avec une cardiopathie, a aussi démontré son efficacité dans l'amélioration de la stéatose hépatique. Dans un essai de 18 mois, sur 278 obèses ou des sujets dyslipidémiques randomisés soit vers un régime méditerranéen (faible en glucides) ou un régime faible en graisses, de meilleures réductions des paramètres de risque cardio- métaboliques chez les sujets suivants le régime méditerranéen étaient associées à une baisse majeure du contenu de graisse hépatique [26].

La chirurgie bariatrique est peut-être l'intervention la plus efficace pour la $\mathrm{NASH}$, avec des améliorations significatives histologiquement et cliniquement documentées [27, 28]. Dans une étude incluant 109 sujets avec une obésité morbide ayant une NASH histologiquement confirmée, à un an de suivi postchirurgical, $85 \%$ des sujets avaient une résolution complète de la NASH [29]. Une autre étude récemment publiée du même groupe, réalisant un suivi sur le long terme des patients avec une NASH ayant subi une chirurgie bariatrique a retrouvé que la réduction de la fibrose suivant la chirurgie survenait de façon progressive, et persistait au minimum 5 ans après [30]. Compte tenu du degré de succès significatif de la chirurgie bariatrique, cette dernière doit être proposée aux candidats remplissant les critères pour cette chirurgie. Par contre, une évaluation pré-thérapeutique multidisciplinaire doit évaluer les patients cirrhotiques au cas par cas dû au risque de complications élevées chez cette catégorie de patients durant et après l'intervention.

L'industrie pharmaceutique a fait des investissements significatifs pour promouvoir les essais thérapeutiques pour la $\mathrm{NASH}$, avec déjà des résultats de phase III disponibles (•Tab. 1). L'industrie est très active sur le développement de médicaments pour la NASH, avec plus de 300 médicaments dans des essais thérapeutiques en 2018, avec un marché destiné aux médicaments approuvés pour le traitement de la NASH estimé entre 20 et 35 milliards de USD par an en 2025 [31]. Selon des nombreuses études longitudinales qui ont démontré que les patients avec un risque intermédiaire ou avancé de fibrose, ont le plus grand risque global et le risque spécifique de mortalité lié à la maladie, ce sous-groupe a été identifié comme la population cible pour

Hier steht eine Anzeige. 


\begin{tabular}{|c|c|c|c|}
\hline Médicament & $\begin{array}{l}\text { Mécanisme } \\
\text { d'action }\end{array}$ & $\begin{array}{l}\text { Statut sur clini- } \\
\text { caltrials.gov (le } \\
\text { 10.07.2020) }\end{array}$ & Résultat phase III (si disponible) \\
\hline Selonsertib & Inhibiteur ASK1 & Terminé & $\begin{array}{l}48 \text { semaines de monothérapie par selonsertib n'ont eu aucun effet antifibrotique chez les } \\
\text { patients atteints de fibrose avancée F3 ou de cirrhose compensée due à la NASH [32] }\end{array}$ \\
\hline Elafibranor & $\begin{array}{l}\text { Agoniste } \\
\text { PPARa/ } \delta\end{array}$ & Actif & $\begin{array}{l}\text { Communiqué de presse en mai } 2020 \text { concernant l'analyse intérimaire de l'essai de phase III: } \\
\text { «l'essai n'a pas atteint le critère principal prédéfini de résolution de la NASH sans aggravati- } \\
\text { on de la fibrose dans la population de } 1070 \text { patients» [33] }\end{array}$ \\
\hline $\begin{array}{l}\text { Resmetirom } \\
\text { (MGL-3196) }\end{array}$ & $\begin{array}{l}\text { Agoniste sélectif } \\
\text { thyroid hormone } \\
\text { receptor- } \beta\end{array}$ & Actif & Résultats de phase III non disponibles \\
\hline Obeticholic acid & $\begin{array}{l}\text { Agoniste farne- } \\
\text { soid } X \text { receptor }\end{array}$ & Actif & $\begin{array}{l}\text { Analyse intérimaire à } 18 \text { mois: } \\
\text { Le critère amélioration de la fibrose sans aggravation de la NASH a été atteint par } 37 \text { (12\%) } \\
\text { patients dans le groupe placebo, } 55(18 \%) \text { dans le groupe acide obéticholique } 10 \mathrm{mg} \\
(p=0,045) \text { et } 71 \text { ( } 23 \%) \text { dans le groupe acide obéticholique } 25 \mathrm{mg}(p=0,0002) . \\
\text { Le critère résolution de la NASH sans aggravation de la fibrose n'a pas été atteint ( } 25 \text { [8\%] } \\
\text { patients dans le groupe placebo, } 35 \text { [11\%] dans le groupe acide obéticholique } 10 \mathrm{mg} \\
[p=0,18] \text { et } 36 \text { [12\%] dans le groupe acide obéticholique } 25 \mathrm{mg}[p=0,13])[34]\end{array}$ \\
\hline Cenicriviroc & $\begin{array}{l}\text { Antagoniste } \\
\text { CCR2/CCR5 }\end{array}$ & Actif & Résultats de phase III non disponibles \\
\hline
\end{tabular}

les médicaments en essai clinique de phase III. Quelques essais commencent déjà à publier les premiers résultats pour la phase III sur la NASH mais certains de ces médicaments ont montré des résultats décevants. Le selonsertib, un inhibiteur ASK1, n’a pas démontré de bénéfices chez les sujets ayant une NASH [32]. Récemment GENFIT a annoncé des résultats décevants d'une analyse intérimaire d'un essai thérapeutique en phase III, testant l'elafibranor, un double agoniste PPAR $\alpha / \delta$ chez l'adulte avec une NASH [33]. Les premiers résultats positifs d'une étude en phase III émanent de l'analyse des résultats à 18 mois de lacide obéticholique, un agoniste FXR [34]. Ce dernier a été administré à une dose journalière de 10 ou $25 \mathrm{mg}$, comparé au placebo, chez des patients avec une biopsie confirmée stade F2-F3 de fibrose. Un des 2 objectifs principaux (amélioration de la fibrose sans aggravation de la NASH) a été atteint chez $23 \%$ chez le groupe qui a reçu $25 \mathrm{mg}$ d'acide obéticholique, comparé à $12 \%$ chez le groupe qui a reçu le placebo alors que l'autre objectif principal (résolution de la NASH sans aggravation de la fibrose) n'a pas été atteint. Cependant, la prise d'acide obéticholique était associée à des effets indésirables, notamment un prurit et une augmentation des taux de LDL cholestérol. Leffet négatif de l'acide obéticholique sur le profil lipidique, res- te un problème particulier vu le risque cardiovasculaire élevé chez les patients avec une NAFLD bien qu'il n'est pas clair si cette élévation est associée à un risque cardiovasculaire augmenté. Cependant, la course pour découvrir de nouveaux médicaments pour la NASH continue.

\section{Conclusion}

La NAFLD est une pathologie à progression lente mais avec une prévalence qui augmente rapidement, des conséquences cliniques significatives et un poids économique et sociétal important. Bien que l'industrie pharmaceutique soit très active dans le domaine de la NASH, jusquà présent aucun traitement pharmacologique n’a été approuvé. L'adoption de la MAFLD, comme nouvelle définition proposée pour la NAFLD avec des critères de diagnostic positifs reste controversée. A l'heure actuelle le clinicien devra en particulier s'assurer d'améliorer le profil de risque cardiovasculaire de ces patients et introduire des mesures hygiéno-diététiques en fonction du stade de sévérité. Par ailleurs, il est évident que en cas de fibrose avancée, voire de cirrhose, le suivi spécialisé s'avère essentiel car le patient devra être candidat à un dépistage du carcinome hépatocellulaire et aux autres complications des hépatopathies avancées.

\section{Take home message}

La stéatopathie dysmétabolique (NAFLD) est la pathologie hépatique la plus fréquente en lien avec l'épidémiologie du diabète et du surpoids. Le dépistage de NAFLD, en particulier ses formes avancées, doit être envisagé chez tous les sujets avec syndrome métabolique. La prise en charge dépend du degré de fibrose mais comprend des mesures hygiénodiététiques, la chirurgie bariatrique chez certains patients et possiblement, dans le futur, une pharmacothérapie ciblée.

\section{Adresse de correspondance

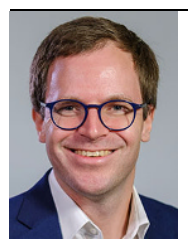 \\ Nicolas Goossens, MD, MSc, PD Service de Gastroentérologie et Hépatologie, Hôpitaux Universitaires de Genève Rue Gabrielle-Perret-Gentil 4, 1205 Genève, Suisse nicolas.goossens@hcuge.ch}

Funding. N. Goossens bénéficie d'un soutien de la ligue Genevoise contre le cancer.

Funding. Open access funding provided by University of Geneva 


\section{Conformité aux directives éthiques}

Conflit d'intérêts L. Zampaglione, J. Ferrari et N.Goossens déclarent ne pas avoir de conflit d'intérêt.

Pour cet article, les auteurs n'ont réalisé aucune étude sur les humains ou les animaux. Les études réalisées étaient conformes aux normes éthiques indiquées dans chaque cas.

Open Access. This article is licensed under a Creative Commons Attribution 4.0 International License, which permits use, sharing, adaptation, distribution and reproduction in any medium or format, as long as you give appropriate credit to the original author(s) and the source, provide a link to the Creative Commons licence, and indicate if changes were made. The images or other third party material in this article are included in the article's Creative Commons licence, unless indicated otherwise in a credit line to the material. If material is not included in the article's Creative Commons licence and your intended use is not permitted by statutory regulation or exceeds the permitted use, you will need to obtain permission directly from the copyright holder. To view a copy of this licence, visit http://creativecommons.org/licenses/by/4.0/.

\section{References}

1. Chalasani N, Younossi Z, Lavine JE et al (2018) The diagnosis and management of nonalcoholic fatty liver disease: Practice guidance from the American Association for the Study of Liver Diseases. Hepatology 67:328-357

2. Caligiuri A, Gentilini A, Marra F (2016) Molecular pathogenesis of NASH. Int JMol Sci 17:1575

3. EASL, EASD, EASO (2016) EASL-EASD-EASO Clinical Practice Guidelines for the management of non-alcoholic fatty liver disease. J Hepatol 64:1388-1402

4. Eslam M, Sanyal AJ, George J et al (2020) MAFLD: a consensus-driven proposed nomenclature for metabolic associated fatty liver disease. Gastroenterology 158:1999-2014.e1

5. Poonawala A, Nair SP, Thuluvath PJ (2000) Prevalence of obesity and diabetes in patients with cryptogenic cirrhosis: a case-control study. Hepatology 32:689-692

6. Younossi ZM et al From NAFLD to MAFLD: Implications of a premature changein terminology. https://aasldpubs.onlinelibrary.wiley.com/doi/ 10.1002/hep.31420.Zugegriffen: 9. Juli 2020

7. Younossi ZM, Koenig AB, Abdelatif D et al (2016) Global epidemiology of nonalcoholic fatty liver disease-meta-analytic assessment of prevalence, incidence, and outcomes. Hepatology 64:73-84

8. Younossi ZM, Stepanova M, Afendy M et al (2011) Changes in the prevalence of the most common causes of chronic liver diseases in the United States from 1988 to 2008. Clin Gastroenterol Hepatol 9:524-530.e1 (quize60)

9. Foschi FG, Bedogni G, Domenicali M et al (2018) Prevalence of and risk factors for fatty liver in the general population of Northern Italy: the Bagnacavallo Study. BMC Gastroenterol 18:177

10. Soresi $M$, Noto $D$, Cefalù $A B$ et al (2013) Nonalcoholic fatty liver and metabolic syndrome in Italy: results from a multicentric study of the Italian Arteriosclerosis society. Acta Diabetol 50:241-249
11. Younossi ZM, Blissett D, Blissett $R$ et al (2016) The economic and clinical burden of nonalcoholic fatty liver disease in the United States and Europe. Hepatology 64:1577-1586

12. United States Centers for Disease Control (CDC) (2020) Stroke facts. https://www.cdc.gov/stroke/ facts.htm (Erstellt: 31. Jan. 2020). Zugegriffen: 14. Apr. 2020

13. Younossi ZM, Stepanova M, Younossi Y et al (2020) Epidemiology of chronic liver diseases in the USA in the past three decades. Gut 69:564-568

14. Younossi ZM, Golabi P, de Avila L et al (2019) The global epidemiology of NAFLD and NASH in patients with type 2 diabetes: a systematic review and meta-analysis. J Hepatol 71:793-801

15. Goossens N, Bellentani S, Cerny A et al (2019) Nonalcoholic fatty liver disease burden - Switzerland 2018-2030. Swiss Med Wkly 149. https://doi.org/ 10.4414/smw.2019.20152

16. Nasr $P$, Ignatova $S$, Kechagias $S$ et al (2017) Natural history of nonalcoholic fatty liver disease: a prospective follow-up study with serial biopsies. Hepatol Commun 2:199-210

17. Singh S, Allen AM, Wang Z et al (2015) Fibrosis progression in nonalcoholic fatty liver vs nonalcoholic steatohepatitis:asystematicreviewand meta-analysis of paired-biopsy studies. Clin Gastroenterol Hepatol 13:643-654.e9 (quize39-40)

18. Ekstedt M, Hagström H, Nasr P et al (2015) Fibrosis stage is the strongest predictor for disease-specific mortality in NAFLD after up to 33 years of followup. Hepatology 61:1547-1554

19. EASL (2015) Non-invasive tests for evaluation of liver disease severity and prognosis. https://easl.eu/ publication/non-invasive-tests-for-evaluationof-liver-disease-severity-and-prognosis/. Zugegriffen: 10 . Juli 2020

20. Tilg H, Moschen AR (2010) Evolution of inflammation in nonalcoholic fatty liver disease: The multiple parallel hits hypothesis. Hepatology 52:1836-1846

21. Petersen KF, Dufour S, Befroy D et al (2005) Reversal of nonalcoholic hepatic steatosis, hepatic insulin resistance, and hyperglycemia by moderate weight reduction in patients with type 2 diabetes. Diabetes 54:603-608

22. Promrat K, Kleiner DE, Niemeier $\mathrm{HM}$ et al (2010) Randomized controlled trial testing the effects of weight loss on nonalcoholic steatohepatitis. Hepatology 51:121-129

23. Vilar-Gomez E, Martinez-Perez Y, CalzadillaBertot $L$ et al (2015) Weight loss through lifestyle modification significantly reduces features of nonalcoholic steatohepatitis. Gastroenterology 149:367-378.e5 (quize 14-15)

24. Adams LA, Harmsen S, St Sauver JL et al (2010) Nonalcoholic fatty liver disease increases risk of death among patients with diabetes: a community-based cohort study. Am J Gastroenterol 105:1567-1573

25. Stepanova M, Younossi ZM (2012) Independent as sociation between nonalcoholic fatty liver disease and cardiovascular disease in the US population. Clin Gastroenterol Hepatol 10:646-650

26. Gepner Y, Shelef I, Komy O et al (2019) The beneficial effects of Mediterranean diet over lowfat diet may be mediated by decreasing hepatic fat content. J Hepatol 71:379-388

27. Lee Y, Doumouras AG, Yu J et al (2019) Complete resolution of nonalcoholic fatty liver disease after Bariatric surgery: a systematic review and meta-analysis. Clin Gastroenterol Hepatol 17:1040-1060.e11
28. Mattar SG, Velcu LM, Rabinovitz M et al (2005) Surgically-induced weight loss significantly improves nonalcoholic fatty liver disease and the metabolic syndrome. Ann Surg 242:610-617 (discussion 618-620)

29. Lassailly G, Caiazzo R, Buob D et al (2015) Bariatric surgery reduces features of nonalcoholic steatohepatitis in morbidly obese patients. Gastroenterology 149:379-388

30. Lassailly G, Caiazzo R, Ntandja-Wandji L-C et al (2020) Bariatric surgery provides long-term resolution of nonalcoholic steatohepatitis and regression of fibrosis. Gastroenterology. https:// doi.org/10.1053/j.gastro.2020.06.006

31. Drew L (2017) Drug development: sprint finish. Nature 551:S86-S89

32. Harrison SA, Wong VW-S, Okanoue T et al (2020) Selonsertib for patients with bridging fibrosis or compensated cirrhosis due to NASH: results from randomized phase III STELLAR trials. J Hepatol 73:26-39

33. GENFIT (2020) GENFIT: announces results from interim analysis of RESOLVE-IT phase 3 trial of elafibranor in adults with $\mathrm{NASH}$ and fibrosis. https://ir.genfit.com/news-releases/ news-release-details/genfit-announces-results interim-analysis-resolve-it-phase-3/. Zugegriffen: 17. Juli 2020

34. Younossi ZM, Ratziu V, Loomba R et al (2019) Obeticholic acid for the treatment of nonalcoholic steatohepatitis: interim analysis from a multicentre, randomised, placebo-controlled phase 3 trial. Lancet 394:2184-2196

35. Younossi ZM, Koenig AB, Abdelatif $D$ et al (2016) Global epidemiology of nonalcoholic fatty liver disease-Meta-analytic assessment of prevalence, incidence, and outcomes. Hepatology 64:73-84

36. Kanwal F, Kramer JR, Mapakshi S et al (2018) Risk of hepatocellular cancer in patients with nonalcoholic fatty liver disease. Gastroenterology 155:1828-1837.e2

Note de l'éditeur. Springer Nature conserve une position neutre en ce qui concerne les revendications juridictionnelles dans les cartes géographiques et les affiliations institutionnelles figurant dans ses publications. 